\title{
THE BANACH-TARSKI PARADOX DICTATES SNYERGISTIC ROUTES FOR SCALE-FREE NEURODYNAMICS
}

Arturo Tozzi (Corresponding author)

Center for Nonlinear Science, University of North Texas

1155 Union Circle, \#311427, Denton, TX 76203-5017 USA

tozziarturo@libero.it

Arturo.Tozzi@unt.edu

James F. Peters

Department of Electrical and Computer Engineering, University of Manitoba

75A Chancellor's Circle, Winnipeg, MB R3T 5V6, Canada and

Department of Mathematics, Adiyaman University, 02040 Adiyaman, Turkey,Department of Mathematics, Faculty of Arts and Sciences, Adiyaman University

02040 Adiyaman, Turkey and Computational Intelligence Laboratory, University of Manitoba, WPG, MB, R3T 5V6, Canada

james.peters3@umanitoba.ca

Neuroscientists are able to detect physical changes in information entropy in available neurodata. However, the information paradigm is inadequate to fully describe nervous dynamics and mental activities such as perception. This paper provides an effort to build explanations to neural dynamics alternative to thermodynamic and information accounts. We recall the Banach-Tarski paradox (BTP), which informally states that, when pieces of a ball are moved and rotated without changing their shape, a synergy between two balls of the same volume is achieved instead of the original one. We show how and why BTP might display this physical and biological synergy meaningfully, making it possible to tackle nervous activities. The anatomical and functional structure of the central nervous system's nodes and edges allows to perform a sequence of moves inside the connectome that doubles the amount of available cortical oscillations. In particular, a BTP-based mechanism permits scale-invariant nervous oscillations to amplify and propagate towards far apart brain areas. Paraphrasing the BPT's definition, we could state that: when a few components of a self-similar nervous oscillation are moved and rotated throughout the cortical connectome, two self-similar oscillations are achieved instead of the original one. Furthermore, based on topological structures, we illustrate how, counterintuitively, the amplification of scale-free oscillations does not require information transfer.

KEYWORDS: Banach-Tarski paradox; brain; power law; fractal; oscillations; information.

The successful information theory permits the appraisal of very broad features of physical/biological systems, suggesting that our world (including ourselves) is made up of a fundamental physical assemblies termed information (Shannon 1948; Bekenstein, 2003). Over time, numerous information-related perspectives have been portrayed: e.g., information theory has been correlated with statistical thermodynamics, Renyi entropy, Bekenstein-Hawking entropy, quantum mechanics, and so on (Jaynes 1957; Lloyd 2000; Marzuoli and Rasetti, 2005, Bromiley et al., 2010; Tozzi et al., 2018). After the launch of the slogan "it from bit" (Wheeler 1990), neuroscientists started to ask themselves whether physical information could be used to describe and quantify brain activity, mental functions and their neural correlates (Tozzi and Peters, 2016). Noteworthy attempts are free-energy Bayesian approaches (Friston 2010) based on energy budgets (Attwell and Laughlin, 2001), and pairwise entropy extracted from fMRI neurodata (Watanabe et al., 2013 and 2014). In particular, neuroscientists provided an effort to correlate modifications in sensations and perceptions with changes in brain informational content, investigating the neural correlates of mental tasks in terms of information entropy. However, it's quite clear that something is amiss with the duet information/nervous activity. Just observing simple pictures and sequences of video frames, it easy to grasp that information entropy is inadequate to assess, describe, quantify and classify the activities and the high-dimensional topological structures of the brain that we term mental functions. The entropic content of different photographs can be accurately quantified through a series of widely available techniques (see, e.g., Allen and Triantaphillidou, 2009). However, a completely grey picture displays zero entropy, while a densely coarsegrained picture with black, grey and white tiny dots displays the highest levels of entropy (Figures 1A-B). From the standpoint of the naïve observer, both the pictures are a collection of meaningless, senseless and devoid of content spots. In turn, photographs depicting, say, a tender infant or a pleasant landscape display values of entropy relatively close to 
each other, despite being equipped with fully different meaning and sense (Figures 1C-D). These pictures produce vivid perceptions that trigger sensations, reasoning, thoughts, i.e., in short, all sorts of psychological activities. This means that observer's sensation is not correlated with the physical informational content of a perceived object. This also means that the efforts of neuroscientists to quantify the changes in brain information entropy during different mental tasks are doomed to failure. Modifications in information detected by the available neuro-techniques (such as, e.g., EEG, fMRI, tractography) are not able to describe mental activities, because psychological functions do not seem to be correlated with physical information. There must be mechanisms other than information that explain how observers perceive objects. Here we propose a mechanism that makes it possible to duplicate brain activities in different areas of the central nervous system, devoid of information transfer. In particular, we show how and why the Banach-Tarski paradox (BPT) permits the replication one of the foremost features of brain oscillations: the occurrence of power laws.
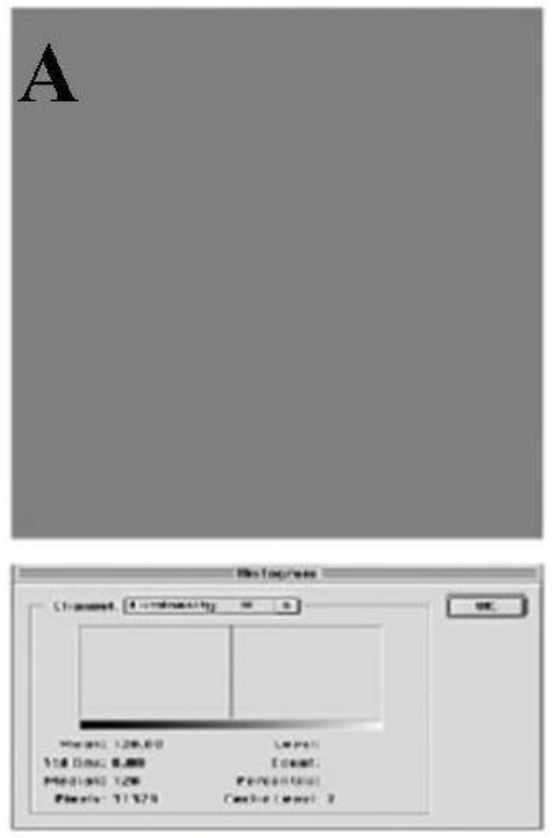

Entropy $=0$
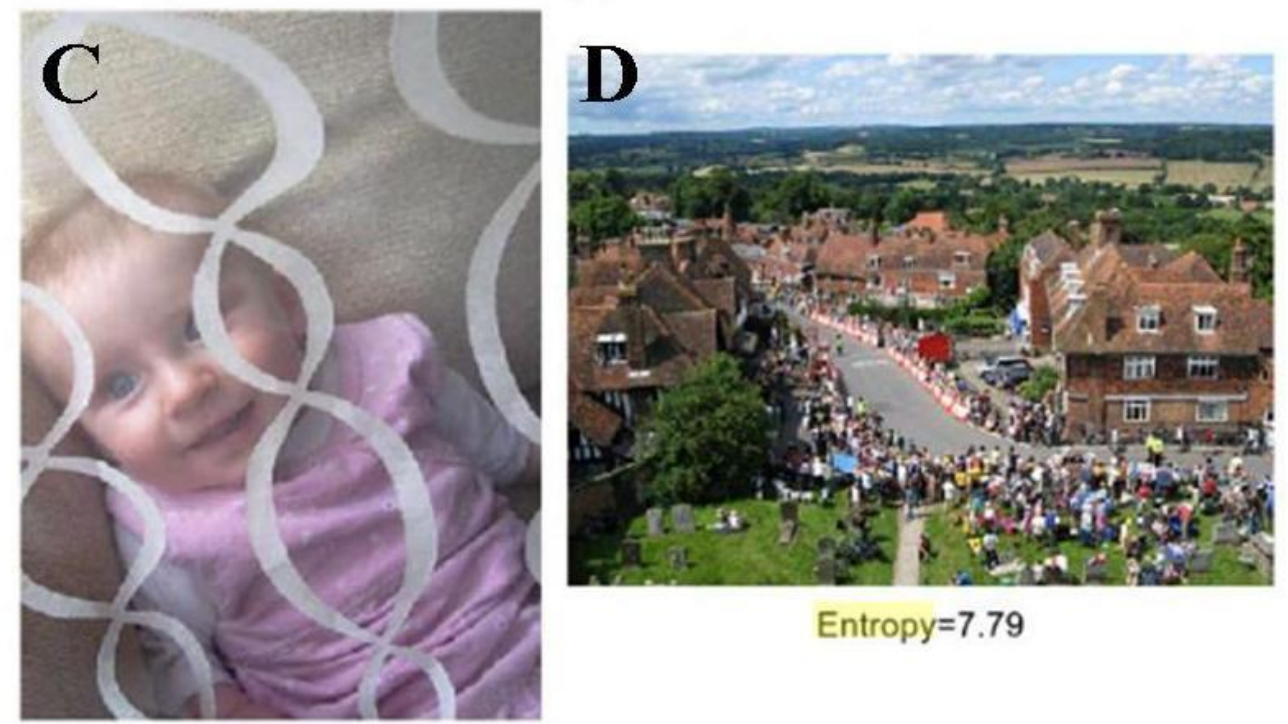

Entropy $=7.79$
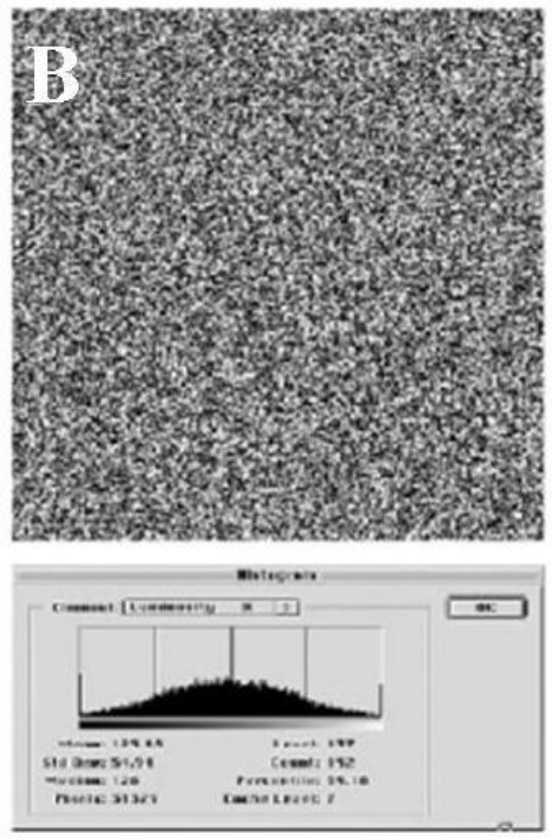

Entropy $=8.0$

Entropy $=7.22$

Figure 1. Entropy level in different pictures. Modified from: Allen and Triantaphillidou (2009). 


\section{A PHYSICAL MEANING FOR THE BANACH TARSKI PARADOX}

The Banach-Tarski paradox (BTP) from set-theoretic geometry suggests that a solid 3- dimensional ball decomposed into at least five disjoint subsets can be reassembled in a different way to yield two identical copies of the original ball (Banach and Tarski, 1924; Churkin 2010). In plain words, when pieces of a ball are moved and rotated without changing their shape, we achieve two balls, instead of the original one. For an example, see Figure 2, that illustrates how a few cuts, followed by simple geometric rotations and translations, allow the duplication of a mathematical structure without volumetric modification. The paradox goes against the everyday experience, because common sense (and physics) presupposes changes in volume when objects are split. Any physical analogy would involve division into two objects with the same volume, but half the density of the original. However, the case of BTP is different: contrary to a physical sphere equipped with a finite number of atoms, the pieces described by BTP are infinitely divisible, i.e., they encompass an infinite number of points. Each piece here is so infinitely complex that it is immeasurable, or, in physical words, it does not have a well-defined measurable volume. These pieces are highly pathological in nature, so that their construction requires the use of the axiom of choice.

In quest for alternatives to information accounts of the brain, here we ask whether BTP could be useful in describing nervous dynamics, despite the adverse premises described above. Our effort is constrained by a few dictates: we need to split a ball (or, in our case, some features of nervous activity) into amorphous, nonmeasurable sets where the volume measure does not count. Does there exist in the brain and the nervous system a feature, a neural assembly, a system, a dynamical process that obeys the conditions and geometric properties dictated by BF?

The answer is affirmative, if we take into account scale-free dynamics. Indeed, when Fourier analysis of brain waves is performed, the frequency spectrum of cerebral activity exhibits a scale-invariant behavior (Mandelbrot 1967; Pritchard 1992):

$\mathrm{S}(\mathrm{f})=1 / \mathrm{f}^{\mathrm{n}}$

where $\mathbf{S}(\mathbf{f})$ is the power spectrum, $\mathbf{f}$ the frequency and $\mathbf{n}$ the power spectral density. The power spectral density, also termed the "fractal dimension", equals the negative slope of a log power versus frequency scatter plot (Milstein et al., 2009). It is noteworthy that fluctuations with complex scaling properties are generated by the brain even in the absence of exogenous perturbations or changes in controlling parameters (Papo 2014). Furthermore, this scale-invariant behavior is not limited to the cortical electric activity, but has also been detected in spontaneous neurotransmitter release (Fox and Raichle, 2007). Indeed, universal scaling seems to stand for a general spatio-temporal property of physiological and pathological brain signals. The emergence of power law distributions has been interpreted in terms of self-organized criticality (Lubeck, 2004): a mechanism of slow energy accumulation and fast energy redistribution drives the system towards a critical state (De Arcangelis and Herrmannn, 2010), so that it has been proposed that cognitive processes can be framed in terms of complex activities equipped with scaling properties (Sengupta and Stemmler, 2014). Power law distributions contain information on how large-scale physiological and pathological outcomes arise from the interactions of many small-scale processes where the volume does not count anymore (Tinker and Velazquez, 2014; Kawe et al., 2019).

Therefore, it is suitable to make use of scale-free oscillations (both "spatial" fractals and/or "temporal" power laws) to build a BTP framework of the nervous activity. Scale-free nervous oscillations display the same features required by the BTP set-theoretic decomposition: these oscillations are nonmeasurable sets with no well-defined volume and absence of mass conservation. Indeed, the occurrence of power laws allows to detect the same oscillatory pattern at countless different levels of observation, independent of the neuronal volume taken into account. Fractals and power laws repeat themselves from the micro- to the macro-levels of observation, so that they are always left unchanged when split and reunited. Paraphrasing the BPT's formulation, we could say that: when at least five components of a scale invariant nervous oscillation are moved, translated and rotated without changing their shape, we achieve two scale invariant nervous oscillations instead of the original one. 

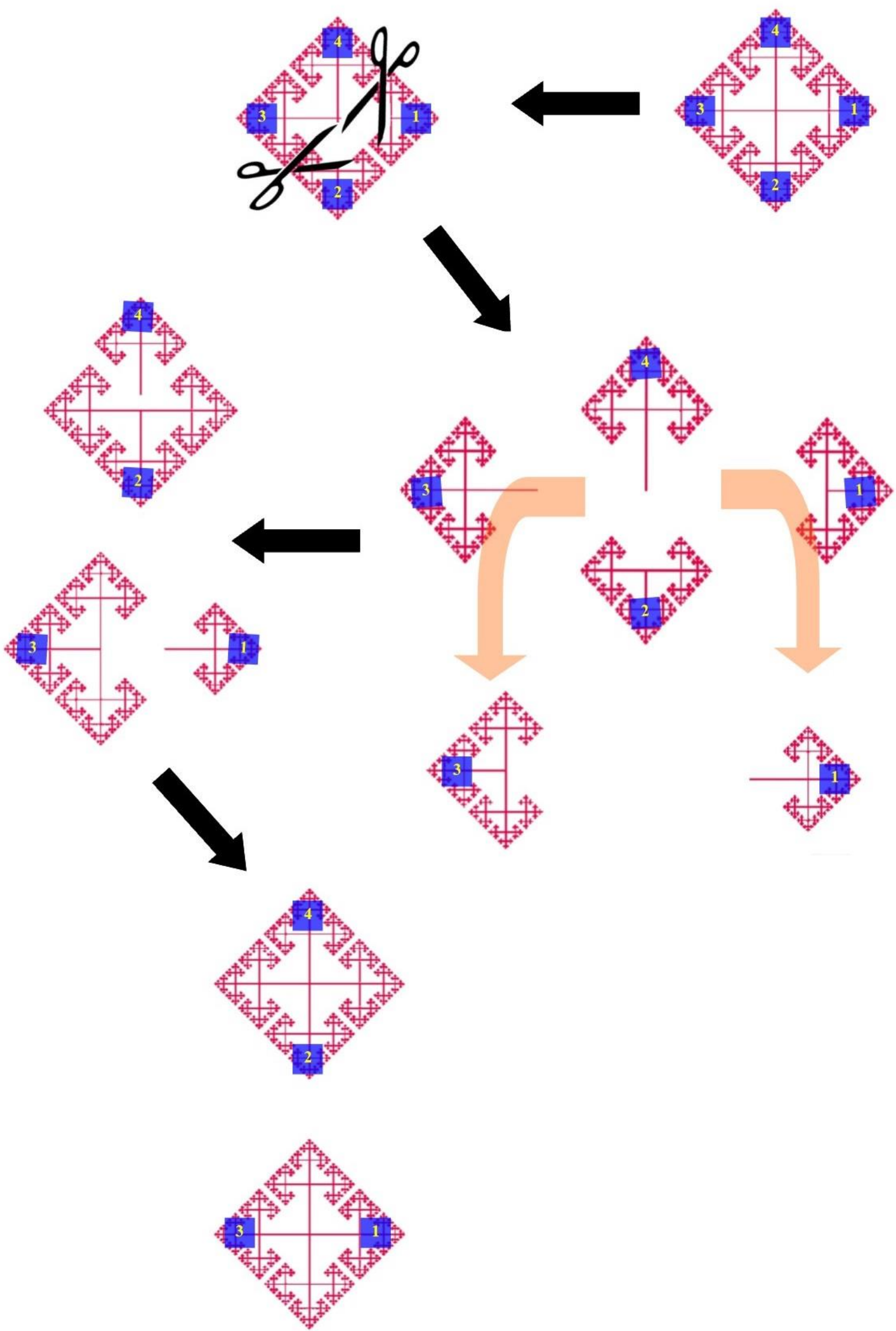

Figure 2. This classical example illustrates the operations required by BTP through decomposition into sets. Starting from a single manifold, once performed a series of cuts and translations, two manifolds are achieved equal to the original one. Note that, if we include the parent (original manifold) as one of the pieces, we get a total of five pieces, that are the minimum amount required to let BTP work. 


\section{POWER LAWS MEET THE BANACH TARSKI PARADOX}

In the previous paragraph, we described theoretical observations to link two apparently irreconcilable frameworks, i.e., the abstract mathematical apparatus of BPT and the real-world scale-free dynamics occurring in the nervous system. Here we provide an effort to operationalize their matching in a real world system: the human brain. In particular, we show how it is feasible to physically/biologically illustrate the counterintuitive claim that objects or processes can be duplicated without changes in volume, energy, information. The canonical example of one sphere becoming two spheres after a series of cuts and rotations/translations has been previously illustrated in Figure 2. Here we describe how, with the choice of just a few cuts, a fractal manifold can be decomposed in three structures with matching description (Figure 3). Technically, BPT allows to achieve $\mathrm{k}$ copies of a ball in the Euclidean $n$-space from one, for any integers $n \geq 3$ and $k \geq$ 1, by using the Lie rotation group $\mathrm{SO}(\mathrm{n})$. In particular, we use the Sierpinski triangle, a fractal fixed set with the overall shape of an equilateral triangle, subdivided recursively into smaller equilateral triangles (Ali et al., 2019). Figure 3 shows how it is allowed to achieve three Sierpinski triangles, starting from just one. Therefore, we can state that, with the proper choice of cuts and translations, scale-free structures obey the BPT dictates.

In order to assess physical and biological dynamics, the paradox can be used also in another slightly different way (Figure 4). In this case, the main issue is the growth of BTP stages, starting with multiple spherical composite vertexes in a nexus that produces multiple sequences of contiguous spacetime spheres (Figures 4A-C). In Figure 4D, BTP is viewed as a piecewise division of one sphere into the interior of another sphere. BTP process starts here with a single sphere serving as a generator of another sphere. In touch with this approach, it is noteworthy that the required pieces are not "solids" in the usual sense, rather infinite scatterings of vertexes (physical counterparts of points in an algebraic topology: see Alexandroff, 1935; Peters, 2020). Such vertexes may stand for minuscule spacetime chunks in shape assemblies that are the basic building blocks of vortex nerve structures in a CW topology (Peters 2018; Peters 2019). Therefore, the multiple spacetime spherical divisions illustrated in Figure 4D lead to incarnation of multiple generators of offspring spheres that are pivots in path-connected vortical structures, which have high affinity to complex-valued activation functions (Ỏzgủr et al., 2020). A path can then be traced from the surface of a parent sphere (call it e1) along the surface of a child sphere (call it e2), stretching in an unbounded fashion to a nexus of contiguous new offspring spheres, forming the collection of BTP generators $\left\{e_{1}, e_{2}, \ldots, e_{k}\right\}, k \rightarrow \infty$. In effect, the algebraic Betti number for a burgeoning BPT sphere is $\beta_{\alpha}=k$ in a free Abelian group representation of a sequence of BTP sphere divisions (Don et al., 2020).

In sum, we achieve the growth of one (Figure 4D) or many (Figure 4A-C) spheres through a well-defined series of stages independent of the volumetric parameter.

The next step is to use this approach to evaluate flows of scale-free nervous oscillations taking place in nervous structures. Indeed, once shown that fractal structures are able to grow and modify according to the BPT dictates, we need to find these fractal structures in the brain. We require two ingredients: a) the scale-free oscillations described in the previous paragraphs; b) a manifold where rotations and translation can be performed. We will use as manifold the node-based correlation matrix of the human brain connectome (Van den Heuvel and Sporns, 2011). Indeed, the anatomical and functional connections described by the connectome are equipped with the proper edges, nodes, angles, bifurcations, and standard reciprocal relationships that allow us to perform the rotations and translations required by BTP. Despite the claim that cortical connections are arranged in self-similar patterns is controversial (see: Sporns 2006 vs. Reese et al. 2012), in this paper we do not require that the connectome is fractal, because we use it just as a static manifold where our operations can take place.

Figure 5 provides a tentative description of how BPT could work, if we consider that brain oscillations are equipped with scale-free temporal dynamics (that correspond to spatial fractals). Indeed, cortical oscillations are equipped with pink noise, i.e., with power law spectrum: therefore, they are self-invariant at different temporal and spatial scales and can be assessed in terms of the growing spheres described above (Figure 5A). Therefore, starting with an oscillation equipped with power law spectrum in a given area of the connectome (Figure 5B, left), we are allowed to perform translation and rotation operations (not shown in the Figure), achieving in other areas of the connectome two (or more) oscillations with the same feature, i.e., with the same power law spectrum (Figure 5B, right). 

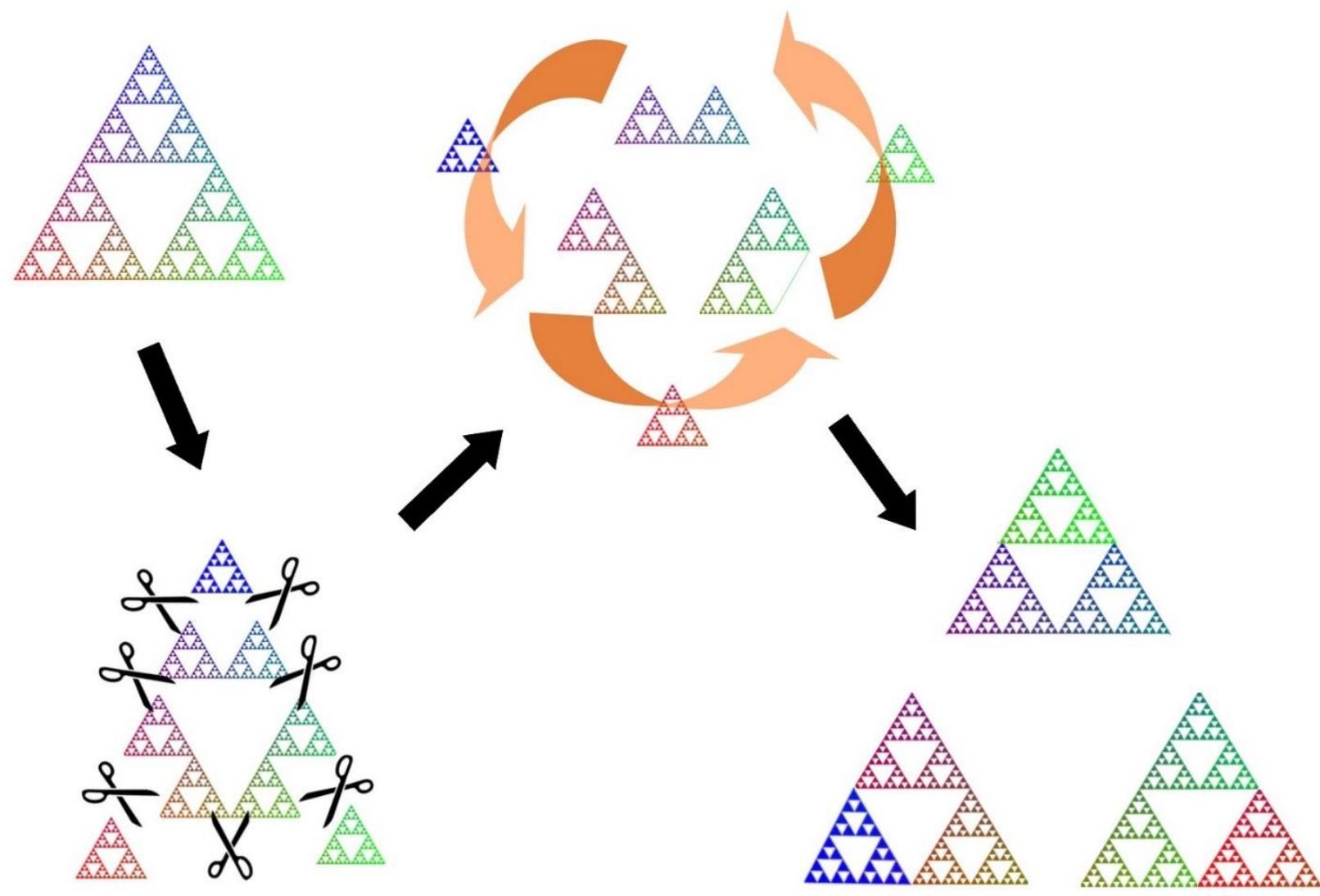

Figure 3. Decomposition in three pieces of a Sierpinski triangle, a fractal manifold made of triangles. 
A

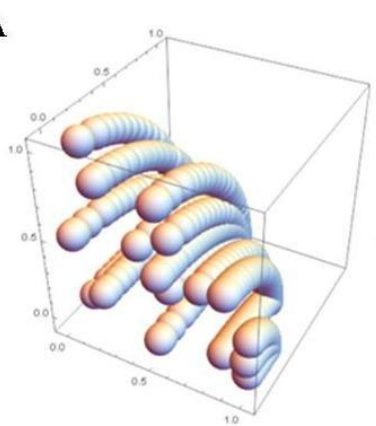

Infant sphere stage

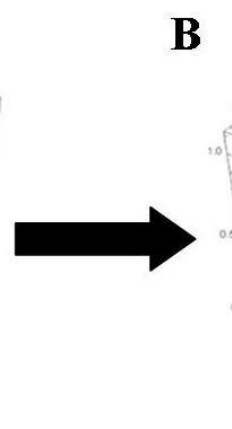

Adolescent sphere stage

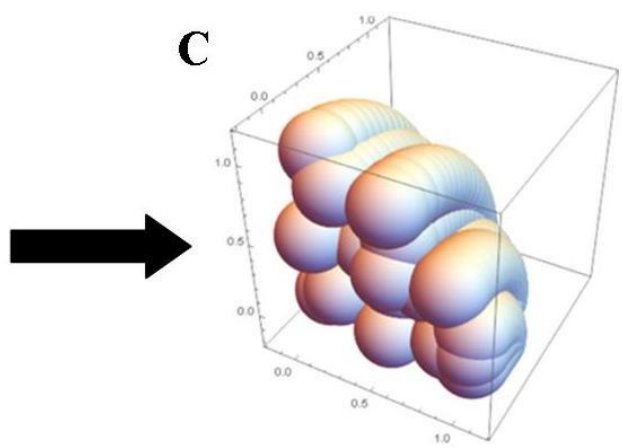

Adult sphere stage

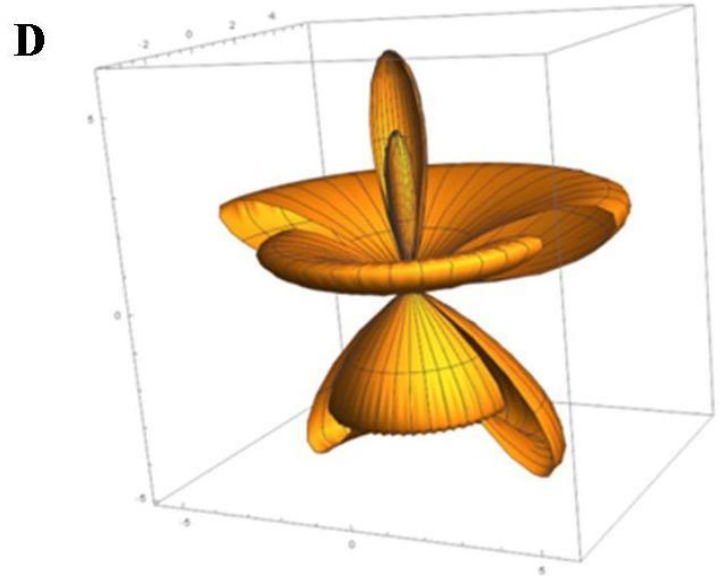

Figure 4. Burgeoning growth BTP stages, starting with multiple tiny spheres (spherical composite vertexes in a nexus) in Figure 4A that continuously form multiple sequences of contiguous spacetime spheres. This beginning BTP stage leads to Figure 4B, where the growth of BTP spherical divisions lead to contiguous adolescent spheres. Next, in a spacetime, seemingly Hegelian synthesis stage, BTP spherical divisions that form path-connected vortexes lead to a nexus of contiguous adult spheres (Figure 4C). Figure 4D: BTP process viewed as a piecewise division of one sphere into the interior of another sphere. In this case, a single sphere serves as a generator of another sphere. 
A
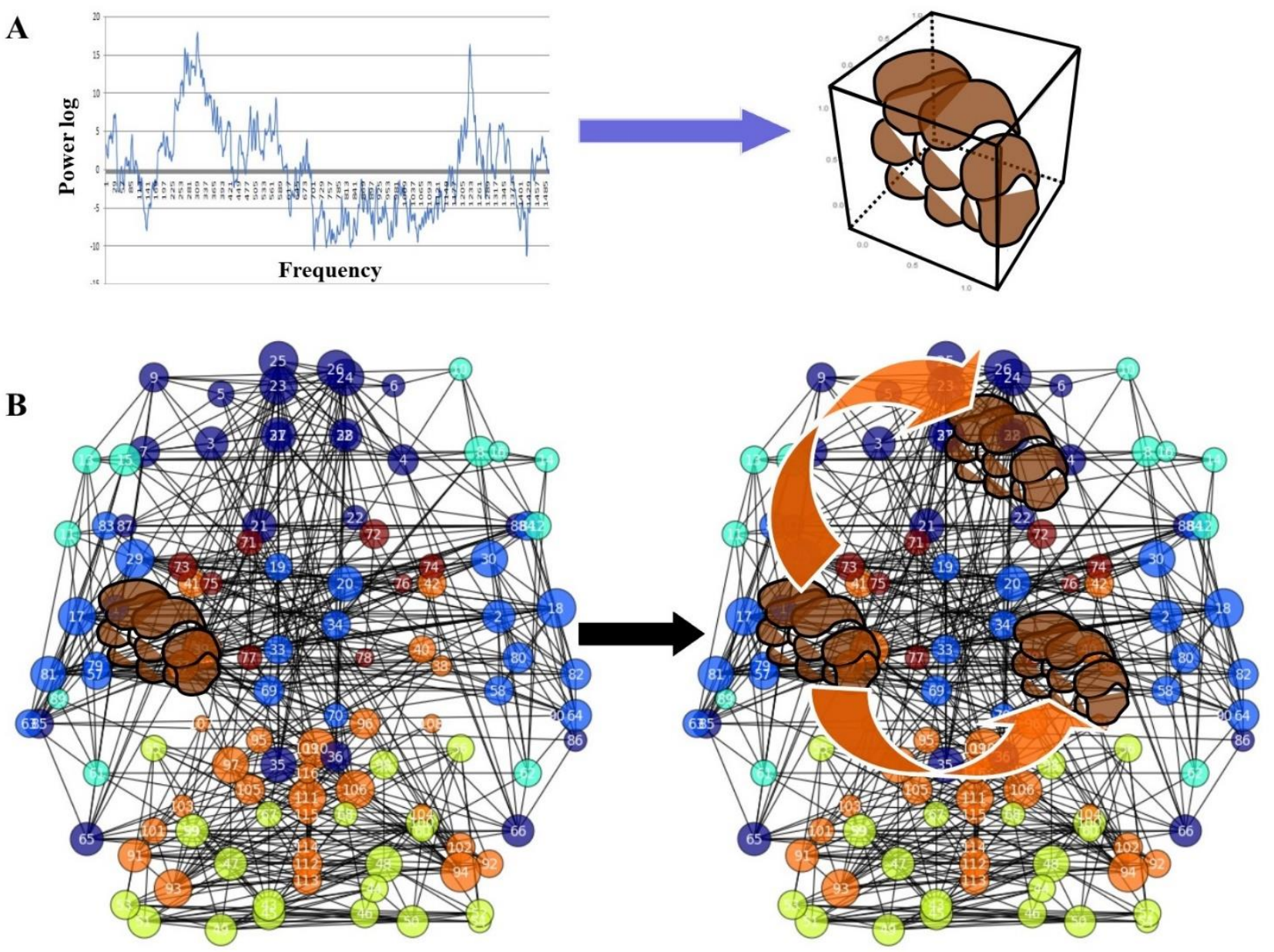

Figure 5. Theoretical example of how BTP might duplicate brain oscillations throughout different cortical areas. Figure 5A depicts the scale-free oscillations experimentally detected in the brain through Fourier analysis and their illustration in terms of multiple tiny spheres. Figure 5B. These scale-free oscillations are located in a single area inside a manifold described in terms of Python/FSL Resting State Pipeline. The pipeline is a collection of steps that can be used to process a single subject's resting state data from raw into a node-based correlation matrix representing connectivity between different regions of the brain. Figure 5C. Here BTT comes into play: with the proper translations and rotations (not shown here) along the edges and the nodes of the pipeline, scale-free oscillations can be split in different chunks with matching description: due to the scale invariant behavior of power law oscillations, every chunk displays the same features of the first one. This allows us to achieve a "duplication" of the original oscillation in two oscillations located in different areas of the connectome. Modified from: https://wiki.biac.duke.edu/biac:analysis:resting_pipeline

\section{CONCLUSIONS}

We described nervous propagation through the Banach Tarski paradox, showing how a sequence of moves related to scale-free brain oscillations allows increases in number of cortical waves. The fact that BTP might work in physical and biological systems would mean, as already suggested by Tozzi and Papo (2020), that topological changes may give rise to modifications devoid of thermodynamic constraints and topological superimposition, so that events may occur that do not require a producing cause. Against all informational odds, it is noteworthy that BPT can be used to describe duplications of nervous oscillations that do not entail information transfer. Although the moves dictated by BPT in a physical/biological setting require a very small amount of energy to be performed, they do not require information transfer. To elucidate this intricate issue, we need to start from topological and symbolic dynamics, where the term "flows" stands for compact metric spaces with groups of homeomorphisms (Furstenberg 1967). In particular, an infinite discrete group G is equipped with an action termed G-flow, made of closed, G-invariant subflows (Glasner et al., 2019). 
To provide an example, take a river, and the local upstream and downstream rush of water: the river stands for the group $\mathrm{G}$, while the two separate rushes of water for subflows. Two dynamical systems or sets (say, our river's proximal and distal subflows), are termed disjoint if at least one of them is minimal, i.e., it is not equipped with further subflows (Glasner and Weiss, 2018). When the subset is minimal, it is devoid of Bernoulli flows, or, in an alternative formulation, the processes with entropy zero are disjoint from Bernoulli flows. In terms of our river, this means there is no input from the preceding spatial proximal subflow to the next iteration, i.e., the distal subflow and, even more important, if the two subsets are disjoint there is no information transfer. It is noteworthy that the "pieces" described by HBT display the same ingredients and features required by disjointed dynamical systems in topological dynamics. Indeed, BPT subflows are related to the geometric study of fixed points of a self-mapping on a metric space. In a geometry of fixed points of a selfmapping on a metric space, Özgür and Taş (2019) established new generalized contractive conditions that ensure a selfmapping having a fixed disc or a fixed circle. In plain terms, we may state that HBT is characterized by an infinite discrete group that is split by an arbitrary free Abelian group of rotation through the axiom of choice, giving rise to at least five subflows. Every different subflow (every "piece") is minimal, and is disjoint from the others. To confirm our suggestion that there is no information transfer among the subflows, it must be emphasized that four of the five sets described in the BTP construction are not Lebesgue-measurable: this means that no information is lost, since the information never existed.

Apart from our suggestions, hints and partial examples referring to BTP can be found in scientific literature. Selforganized pattern behavior is ubiquitous throughout nature, from fish schooling to collective cell dynamics during organism development. McGuirl et al. (2020) quantified skin pattern formation in of wild-type and mutant zebrafish, introducing methods based on topological data analysis and interpretable machine learning for quantifying both agentlevel features and global pattern attributes on a large scale. In particular, they showed that persistent homology allows to proceed from noisy data to corresponding manifold expansions (see Figure 2 of their paper). This expansion, sampled from a figure-eight shape that progressively becomes less fuzzy and increased in size and mass, reminds the pseudovolumetric increases prescribed by BTP. To provide another example, Peron et al. (2020) assessed computational modelling, imaging and single-cell ablation in layer $2 / 3$ of the mouse vibrissal somatosensory cortex, showing that recurrent activity in cortical neurons can drive input-specific amplification during behavior. We suggest that the operations required by BPT are guaranteed by local recurrent circuits and spatially intermingled neurons with similar receptive fields/increased connectivity that could implement amplification, allowing pattern completion and other computations. In turn, Borzenets et al. (2020) investigated the spatial distribution of exotic non-Fermi liquids formed by multiple magnetic impurities. When a magnetic impurity exists in a metal, conduction electrons form a spin cloud. This phenomenon, termed Kondo effect, allows the production of a quantum coherent spin-screening cloud that forms spinsinglet entanglement with the impurity. In touch with our framework of BPT propagation, the Kondo cloud can spread out over several micrometres, although the spins interact just locally around the impurity.

The last, but not the least, the recently described brain vortexes and Betti numbers in cortical lag threads (Don et al., 2020) can be duplicated with simple spatial transformations (the passage of oscillations through brain wires), without the use of energy transfer. This is a fruitful operation, because allows to introduce a new form of Borsuk-Ulam theorem (BUT) in terms of Betti numbers too. In fact, the underlying mechanism in instantiations of BUT (Tozzi and Peters, 2017) provides a terse description of the inner workings of BTP in the context of neuro-dynamics. Here are examples: a) Antipodal, symmetric vortexes serving as generators of offspring spheres dictated by BPT on the surface of a sphere have the same algebraic Betti number (count of the number of generators), with an underlying geometric Betti number (e.g., count of the number of cycles in each vortex). This form of BUT carries over into the study of symmetric activation regions in rsFMRI videos; b) BPT-produced antipodal, symmetric vortexes on the surface of a sphere have the same algebraic Betti number (count of the number bridge segments $\mathrm{x} 2$ attached between vortex cycles). Therefore, a BPT-based approach allows to tackle brain issues from an unusual standpoint.

\section{REFERENCES}

1) Alexandroff P. 1932, 1961. Elementary Concepts of Topology. Dover Pubs., Inc., New York. ISBN 0-48660747-X.

2) Ali A, Rafique H, Arshad T, Alqarni MA, Chauhdary SH, Bashir AK. 2019. A Fractal-Based Authentication Technique Using Sierpinski Triangles in Smart Devices. Sensors (Basel), 19(3). pii: E678. doi: $10.3390 / \mathrm{s} 19030678$.

3) Allen E, Triantaphillidou S. 2009. The Manual of Photography. Tenth Edition. Taylor and Francis Group, Abdingdon, Oxon.

4) Attwell D, Laughlin SB. 2001. An energy budget for signaling in the grey matter of the brain. Journal of Cerebral Blood Flow and Metabolism: Official Journal of the International Society of Cerebral Blood Flow and Metabolism, 21(10):1133-1145. http://doi.org/10.1097/00004647-200110000-00001. 
5) Banach S, Tarski A. 1924. Sur la décomposition des ensembles de points en parties respectivement congruentes. Fundamenta Mathematicae, 6: 244-277.

6) Bekenstein JD. 2003. Black holes and information theory. arXiv:quant-ph/0311049.

7) Borzenets I, Shim J, Chen JCH, Ludwig A, Wieck AD, et. al. 2020. Observation of the Kondo screening cloud. Nature. 2020 Mar;579(7798):210-213. doi: 10.1038/s41586-020-2058-6.

8) Bromiley PA, Thacker NA, Bouhova-Thacker E. 2010. Shannon entropy, Renyi entropy, and information. Tina 2004-004, Statistic and Inf Series, Imaging Sci. and Biomed. Eng., Univ. of Manchester, UK.

9) Churkin VA. 2010. A continuous version of the Hausdorff-Banach-Tarski paradox. Algebra and Logic. 49 (1): 81-89. doi:10.1007/s10469-010-9080-y.

10) de Arcangelis L, Herrmann HJ. 2010. Learning as a phenomenon occurring in a critical state. Proceedings of the National Academy of Sciences 107, 3977-3981.

11) Don AP, Peters JF, Ramanna S, Tozzi A. 2020. Topological View of Flows inside the BOLD Spontaneous Activity of the Human Brain. Front. Comput. Neurosci. | doi: 10.3389/fncom.2020.00034.

12) Fox MD, Raichle ME. 2007. Spontaneous fluctuations in brain activity observed with functional magnetic resonance imaging. Nature reviews. Neuroscience 8, 700-711.

13) Friston K. 2010. The free-energy principle: a unified brain theory? Nature Reviews Neuroscience, 11(2), 127138. http://doi.org/10.1038/nrn2787.

14) Furstenberg H. 1967. Disjointness in ergodic theory, minimal sets, and a problem in diophantine approximation. Mathematical systems theory, 1:1-49.

15) Glasner E, Weiss B. 2018. On the disjointness property of groups and a conjecture of Furstenberg. arXiv: 1807.08493.

16) Glasner E, Tsankov T, Weiss B, Zucker A. 2019. Bernoulli disjointness. arXiv:1901.03406.

17) Jaynes ET. 1957. Information Theory and Statistical Mechanics. Phys. Rev 106: 620.

18) Kantor FW. 1977. Information Mechanics. Wiley-Interscience. ISBN 10: 0471029688 / ISBN 13: 9780471029687.

19) Kawe TNJ, Shadli SM, McNaughton N. 2019. Higuchi's fractal dimension, but not frontal or posterior alpha asymmetry, predicts PID-5 anxiousness more than depressivity. Sci Rep, 9(1):19666. doi: 10.1038/s41598-01956229-w.

20) Lloyd S. 2000. Ultimate physical limits to computation. Nature 406, 1047-1054 (31 August 2000) doi: $10.1038 / 35023282$

21) Lübeck, S. 2004. Universal scaling behavior of non-equilibrium phase transitions. International Journal of Modern Physics B 18, 3977-4118.

22) Mandelbrot B. 1967. How long is the coast of Britain? Statistical self-similarity and fractional dimension. Science, 56(3775):636-8.

23) Marzuoli A, Rasetti M. 2005. Computing Spin Networks. Annals of Physics 318: 345-407.

24) McGuirl MR, Volkening A, Sandstede B. 2020. Topological data analysis of zebrafish patterns. Proc Natl AcadSci U S A. 2020 Mar 10;117(10):5113-5124. doi: 10.1073/pnas.1917763117.

25) Milstein J, Mormann F, Fried I, Koch C. 2009. Neuronal shot noise and Brownian 1/f2 behavior in the local field potential. PLoS One. 2009;4(2):e4338. doi: 10.1371/journal.pone.0004338.

26) Ỏzgủr N, Taş N, Peters JF. 2020. New complex-valued activation functions. An Int. J. of Optimization and Control Theories \& Applications 10(1), 66-72, http://doi.org/10.11121/ijocta.01.2020.00756.

27) Özgür N, Taş N. 2019. Pata Zamfirescu Type Fixed-Disc Results with a Proximal Application. arXiv:1910.12302.

28) Papo D. 2014. Functional significance of complex fluctuations in brain activity: from resting state to cognitive neuroscience. Frontiers in systems neuroscience 8, 112

29) Peron S, Pancholi R, Voelcker B, Wittenbach JD, Ólafsdóttir HF, et al. 2020. Recurrent interactions in local cortical circuits. Nature; 579(7798):256-259. doi: 10.1038/s41586-020-2062-x. Epub 2020 Mar 4.

30) Peters JF. 2018. Proximal vortex cycles and vortex nerves. Non-concentric, nesting, possibly overlapping homology cell complexes, J. of Mathematical Sciences and Modelling 1(2), 80-85, arXiv:1805.03998.

31) Peters JF. 2019. Vortex nerves and their proximities. Nerve Betti numbers and descriptive proximity. Bull. of the Allahabad Math. Soc. 34(2), 263-276.

32) Peters JF. 2020. Computational Geometry, Topology and Physics of Digital Images with Applications. Shape Complexes, Optical Vortex Nerves and Proximities. Springer Nature, Switzerland AG, ISBN 978-3-030-221911.

33) Pritchard WS. 1992. The brain in fractal time: 1/f-like power spectrum scaling of the human electroencephalogram. The International journal of neuroscience 66, 119-129.

34) Reese TM, Brzoska A, Yott DT, Kelleher DJ. 2012. Analyzing Self-Similar and Fractal Properties of the C. elegans Neural Network. PLoS ONE 7(10): e40483. (doi:10.1371/journal.pone.0040483).

35) Sengupta B, Stemmler MB. 2014. Power Consumption During Neuronal Computation. Proceedings of the IEEE 102, 738-750.

36) Shannon CE. 1948. A Mathematical Theory of information. The Bell System Technical Journal 27:379-423. 
37) Sporns O. 2006. Small-world connectivity, motif composition, and complexity of fractal neuronal connections. Biosystems 85(1):55-64. https://doi.org/10.1016/j.biosystems.2006.02.008.

38) Tinker J, Velazquez JL. 2014. Power law scaling in synchronization of brain signals depends on cognitive load. Front Syst Neurosci. 2014 May 1;8:73. doi: 10.3389/fnsys.2014.00073.

39) Tozzi A, Peters JF. 2016. A Topological Approach Unveils System Invariances and Broken Symmetries in the Brain. Journal of Neuroscience Research 94 (5): 351-65. doi:10.1002/jnr.23720.

40) Tozzi A, Peters JF. 2017. From abstract topology to real thermodynamic brain activity. Cogn Neurodyn. 11(3):283-292. doi: 10.1007/s11571-017-9431-7.

41) Tozzi A, Peters JF, Cankaya MN. 2018. The informational entropy endowed in cortical oscillations. Cognitive Neurodynamics, 12(5), 501-507. DOI: 10.1007/s11571-018-9491-3.

42) Tozzi A, Papo D. 2020. Projective mechanisms subtending real world phenomena wipe away cause effect relationships. Progress in Biophysics and Molecular Biology. 151:1-13. DOI: 10.1016/j.pbiomolbio.2019.12.002.

43) Van den Heuvel MP, Sporns O. 2011 Rich-club organization of the human connectome. J Neurosci. 31, 1577515786. doi: 10.1523/JNEUROSCI.3539-11.2011.

44) Wheeler JA. 1990. Information, physics, quantum: The search for links. In Zurek, Wojciech Hubert. Complexity, Entropy, and the Physics of Information. Redwood City, California: Addison-Wesley. ISBN 9780201515091. OCLC 21482771.

45) Watanabe T, Hirose S, Wada H, Imai Y, Machida T, et al. 2013. A pairwise maximum entropy model accurately describes resting-state human brain networks. Nature Communications, 4, 1370. http://doi.org/10.1038/ncomms2388.

46) Watanabe T, Kan S, Koike T, Misaki M, Konishi S, et al. 2014. Network-dependent modulation of brain activity during sleep. NeuroImage, 98, 1-10. http://doi.org/10.1016/j.neuroimage.2014.04.079. 